\title{
Design of artificial neural networks using a genetic algorithm to predict saturates of vacuum gas oil
}

\author{
Dong Xiucheng ${ }^{1 *}$, Wang Shouchun ${ }^{1}$, Sun Renjin ${ }^{1}$ and Zhao Suoqi ${ }^{2}$ \\ ${ }^{1}$ School of Business Administration, China University of Petroleum, Beijing 102249, China \\ ${ }^{2}$ School of Chemical Science and Engineering, China University of Petroleum, Beijing 102249, China \\ (C) China University of Petroleum (Beijing) and Springer-Verlag Berlin Heidelberg 2010
}

\begin{abstract}
Accurate prediction of chemical composition of vacuum gas oil (VGO) is essential for the routine operation of refineries. In this work, a new approach for auto-design of artificial neural networks (ANN) based on a genetic algorithm (GA) is developed for predicting VGO saturates. The number of neurons in the hidden layer, the momentum and the learning rates are determined by using the genetic algorithm. The inputs for the artificial neural networks model are five physical properties, namely, average boiling point, density, molecular weight, viscosity and refractive index. It is verified that the genetic algorithm could find the optimal structural parameters and training parameters of ANN. In addition, an artificial neural networks model based on a genetic algorithm was tested and the results indicated that the VGO saturates can be efficiently predicted. Compared with conventional artificial neural networks models, this approach can improve the prediction accuracy.
\end{abstract}

Key words: Saturates, vacuum gas oil, prediction, artificial neural networks, genetic algorithm

\section{Introduction}

Vacuum gas oil (VGO) is the important fraction of crude oil and the main feedstock for the catalytic cracking process. The hydrocarbon group-type compositions and structural groups of VGO are the fundamental data for engineering design and optimization of processing, and they play an important role in the procedure analyzing of secondary processing, engineering calculation and process modeling. The hydrocarbon-type of petroleum fractions can be obtained by the gas chromatography (GC). However, this technique has proven time-consuming and costly because of the need for specific equipment. Hence a lot of research is aimed at deriving relationships between the physical properties and hydrocarbon group-type compositions of petroleum fractions.

Empirical methods were widely used in early studies. For example, Nes and Westen (1951) developed the n-d-M method to determine the structural groups of the vacuum fractions by using the refractive index $(n)$, density $(d)$ and molecular weight $(M)$. Waterman et al (1958) used a simple graphical method to correlate the physical properties with the chemical structure of the mineral oil. Riazi and Daubert (1980) built correlations based on the factors: viscosity gravity function (VGF), Viscosity-Gravity Constant (VGC) and refractivity intercept, which is the refractive index minus half the density. Riazi and Daubert (1986) used former factors and new factors such as: carbon to hydrogen weight

*Corresponding author. email: dongxiucheng@yahoo.com.cn Received March 16, 2009 ratio and molecular weight. The correlations of Nwadinigwe and Okoroji (1990) are based on refractivity-intercept. The correlations of El-Hadi and Bezzina (2005) are based on normal boiling point, density, refractive index and carbon to hydrogen weight ratio. But the correlations between the hydrocarbon-type and the physical properties of petroleum fractions are complex and non-linear, and these methods are found to give less accurate results. In recent studies, Xing and Cheng (2006) developed linear programming using a least squares algorithm to correlate the VGO hydrocarbon-type with its physical properties. Liu et al (2007) built a regression model to predict the hydrocarbon compositions of VGO based on five conventional physical properties of VGO, namely density, refractive index, molecular weight, sulfur content and carbon-hydrogen ratio. Sun et al (2008) used an artificial neural network (ANN) to predict the saturates of sour vacuum gas oil based on average boiling point, density, molecular weight and refractive index and obtain good results.

In spite of the successful applications in various areas, neural networks are usually designed through iterative trialand-error approaches and no general framework is used to find the optimum structure of a neural network for a specific task. Network design mainly depends on the designer's experience. Therefore, it is a time-consuming and tedious process.

In this study, a new method is proposed to improve the prediction of VGO saturates by optimizing the network scenarios through coupling a multilayer feedforward network with a genetic algorithm (GA). The number of neurons in the hidden layer, the momentum and the learning rate are 
determined by using GA. In other words, a novel artificial neural networks model based on a genetic algorithm is developed to build relationship between VGO saturates and the factors as average boiling point, density, molecular weight, viscosity and refractive index.

\section{Artificial neural networks and genetic algorithm}

\subsection{Artificial neural networks}

An artificial neural network is a highly simplified model of the biological structures found in a human brain. Three elements are particularly important in any model of artificial neural networks: the structure of the nodes, the topology of the network and the learning algorithm used to find the weights of the network (Rojas, 1996).

In terms of their topology structures, neural networks can be divided into two types: feedforward networks and recurrent networks. There are two types of learning algorithm used in neural networks: supervised and unsupervised learning. Different types of networks use different types of learning algorithm.

Among ANN models, the back-propagation (BP) neural network is the most well-known one. The steepest descent method is used as the learning rule of BP networks, with this method, the errors of the output of the BP network are propagated back to adjust the weights of interconnections to minimize the total error.

An ANN application has to go through three phases. In the first phase, the ANN is trained by using a subset of the input-output dataset. While in the second phase, the trained network is cross validated to prevent over training(over generalization) by using another subset of the input-output dataset. In the third phase, the ANN is tested by using a third subset of dataset to evaluate its prediction (generalization) performance.

ANN can discover patterns adaptively from the data. The ANN is capable of learning complex relationships from many individual examples (or experiences). Compared with conventional algorithms, artificial neural networks are good at dealing with complex nonlinear problems. They have fault and noise tolerance and in addition, high robustness and generalization capability.

Due to these properties, an ANN is well suited to forecasting. Empirically, numerous successful applications have been achieved in forecasting.

\subsection{Genetic algorithms}

A Genetic Algorithm (GA) is considered to be a heuristic and stochastic optimization technique based on evolution theory and genetic principles. It is a rapidly growing area of artificial intelligence. GA was introduced by Holland (1975) and further developed by Goldberg (1989) and Michalewicz (1996).

Compared with other search techniques, GA is easy to use because it does not require derivative information or definite initial estimates in the solution space. Genetic Algorithms start to search from multiple points in the solution space to have a higher probability of finding the global optimal solution.

A Genetic Algorithm is composed of the selection, crossover and mutation operators. The selection operator chooses excellent individuals from the current population for reproduction based on individual fitness to the environment. The selection achieves Darwin's principle of survival of the fittest.

The crossover operator combines the features of two parent chromosomes to form two similar offspring by swapping corresponding segments of the parents (Goldberg, 1989). The purpose of crossover is that future generations can inherit better genetic information.

The mutation operator arbitrarily alters one or more genes of a selected chromosome, by a random change with a probability equal to the mutation rate. The mutation operator introduces some extra variability into the population to maintain diversity of the population and prevent premature convergence (Goldberg, 1989). Mutation generally prevents the GA from falling into local extremes.

The fitness function of chromosomes is defined as follows:

$$
F=\frac{1}{E(\eta, \alpha, s)+\xi}
$$

where $\xi$ is a minor positive quantity, $\mathrm{E}(\eta, \alpha, \mathrm{s})$ is the error function of neural network which is defined as follows,

$$
E(\eta, \alpha, s)=\frac{1}{2} \sum_{p=1}^{n} \sum_{j=1}^{m}\left(t_{j}(p)-y_{j}(p)\right)^{2}
$$

where $t_{j}(p)$ and $y_{j}(p)$ are the desired output and actual output of the network at the pth sample point, respectively; $n$ and $m$ are the number of training samples and the number of neurons in the output layer, respectively.

$F$ is the function of the initial momentum, learning rate and the number of nodes in the hidden layer. Following the experimental formula, the scope for the number of nodes in the hidden layer is determined and $0<\alpha, \eta<1$. The three factors are encoded into a chromosome, that is, as an individual in the population. The steps of genetic algorithm for finding the optimal solution are given as:

(1) In accordance with the real-coded rules, randomly generated individuals constitute the initial population and each individual is an initial solution to the problem. The fitness value of each individual is evaluated according to fitness function.

(2) Using the fitness value, roulette-wheel selection algorithm is applied to choose chromosomes. The higher the fitness, the greater the probability that the chromosome enters the next generation.

(3) One-point crossover operation is carried out to produce new chromosomes according to the crossover probability.

(4) Uniform mutation operation is carried out to produce new chromosomes according to the mutation probability.

(5) Repeat steps 2-4 until the maximum generation. 
After the evolution of the population is finished, the GA converges to produce the best chromosome(s), which represents the optimal or near-optimal solution to the problem under study. This operation flowchart is shown in Fig. 1.

\section{Predicting model based on ANN-GA}

\subsection{Data definitions and sources}

From petrochemistry we know that the important influencing factors on saturates of vacuum gas oil are the average boiling point, density, molecular weight, viscosity, refractive index, hydrogen-carbon ratio and carbon residue.
Based on available experimental data, five physical properties, namely average boiling point, density, molecular weight, refractive index and viscosity, are selected as input variables to predict saturates. Choice of samples usually follows the principles that sample data are adequate, representative and uniformly distributed. All the selected influencing factors are summarized in Table 1.

The sample data are chosen from 170 kinds of vacuum gas oil at home and abroad and 56 kinds of analysis data of VGO are selected for use. In general, $90 \%$ of the data are used for training and cross-validation and the other $10 \%$ data are used for testing.

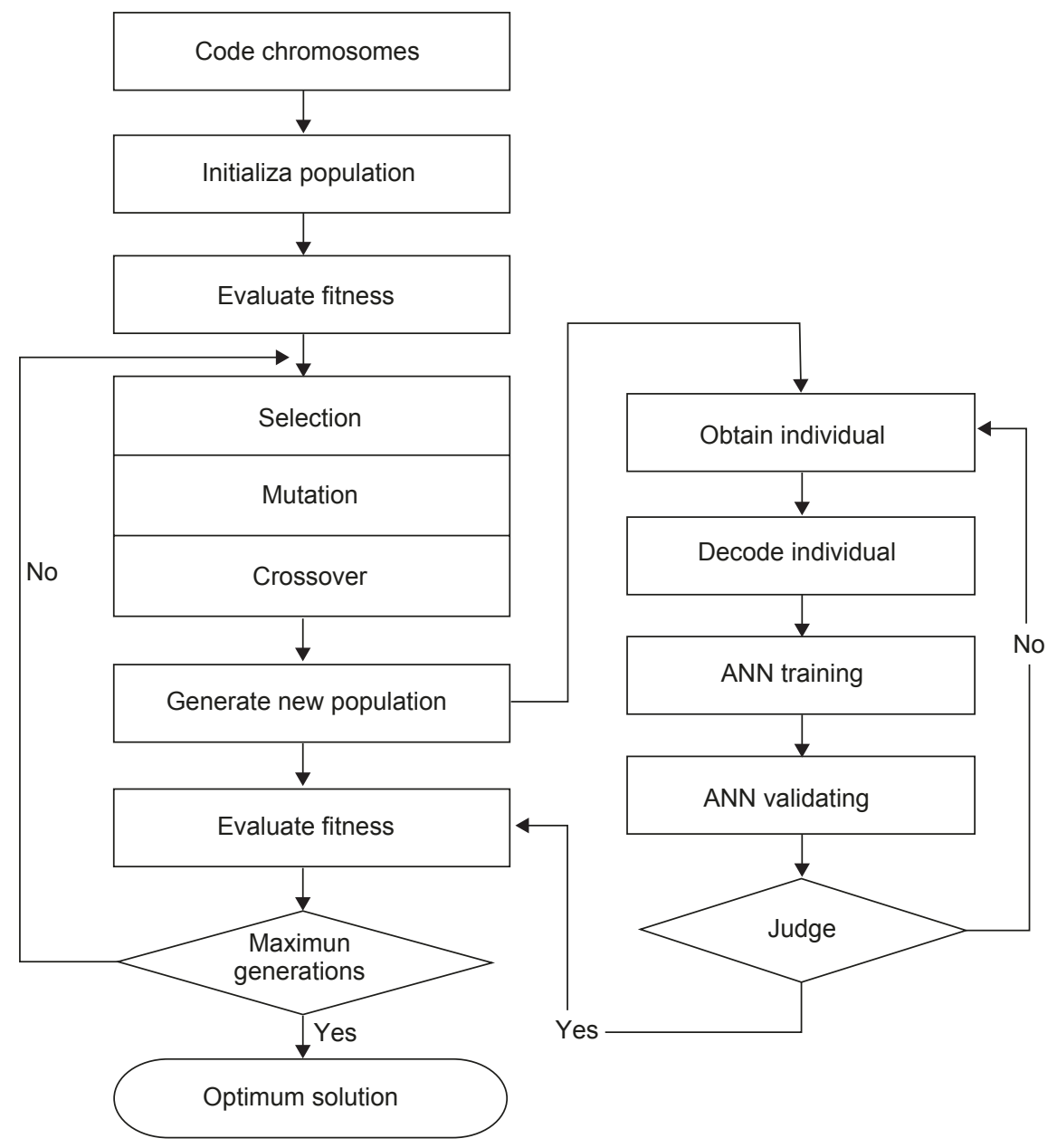

Fig. 1 The flowchart of the optimization process of GA

Table 1 Summary of Input variables

\begin{tabular}{ccc}
\hline Factors & Symbol & Unit \\
Density & $T_{\mathrm{b}}$ & ${ }^{\circ} \mathrm{C}$ \\
Molecular weight & $D$ & $\mathrm{~g} / \mathrm{cm}^{3}$ \\
Viscosity & $M$ & \\
Refractive index & $\eta$ & pascal second \\
Saturates & $n$ & \\
\hline
\end{tabular}

\subsection{ANN-GA model scheme}

The back propagation (BP) algorithm is a well-known method for training multilayer feedforward artificial neural networks (FFANNs). Although the BP algorithm is successful, it has some disadvantages. The algorithm is not guaranteed to find the global minimum of error space and the convergence tends to be extremely slow. In addition, the selection of the learning factor and momentum factor, which is usually determined by experience, also affects the convergence of the BP neural network. In the present work, the number of neurons in the hidden layer, and the momentum and the learning rates are determined by using the genetic algorithm.

The number of hidden layers and the number of nodes in 
each hidden layer depend on the complexity of the patterns and the nature of the problem to be solved. The use of a single hidden layer is sufficient to approximate to any continuous function as closely as requested (Funahashi, 1989; Hornik et al, 1990), and Patuwo et al (1993) reported that having more than three layers may not result in significant performance improvements. Thus, in our study, a three-layer ANN is used.

These five influencing factors are used as inputs to the ANN-GA network and the saturates of VGO as an output. The three-layered ANN-GA network used in this work has 5-10-1 structure, which consists of 1 input layer, 1 hidden layer and 1 output layer. The number of neurons in the input layer is 5 and there is one neuron in the output layer which is VGO saturates. The number of neurons in the hidden layer obtained by GA is 10 . The design of the ANN-GA is shown in Fig. 2. The tanh function is used for each node of the hidden layer and for the output layer as the transfer functions in the ANN-GA model.

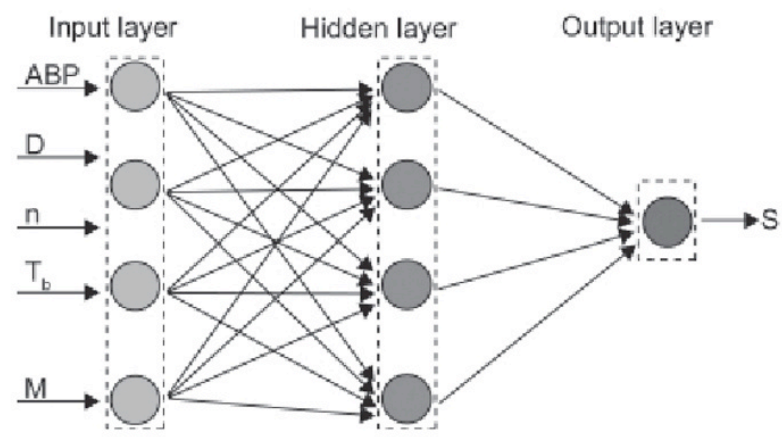

Fig. 2 The structure of a three-layer feedforward artificial neural network

In this study, roulette-wheel for selection, one-point for crossover and uniform for mutation operators are applied to train the networks. The parameters used for searching for the optimal conditions are summarized in Table 2. It is found that the optimum number of neurons in the hidden layer for networks is 10 .

Table 2 Parameter values for GA

\begin{tabular}{cc}
\hline Parameter & Value \\
\hline Population size & 30 \\
Maximum generations & 20 \\
Probability crossover & 0.85 \\
Probability mutation & 0.01 \\
\hline
\end{tabular}

Like the GA method, the trial-and-error method is used to train the ANN models by changing the number of neurons in the hidden layer from 2 to 20 . Table 3 shows the results of the trial-and-error method and the GA method and from Table 3 we find that the GA method is better than the trial-and-error method.
Table 3 Results of the trial-and-error method and its comparison to the GA method

\begin{tabular}{ccccc}
\hline Model & Number of neurons & $r$ & NMSE & MAE \\
\hline ANN-GA & 8 & 0.935 & 0.152 & 2.59 \\
ANN & 10 & 0.891 & 0.382 & 4.32 \\
\hline
\end{tabular}

Notes: $r$-linear correlation coefficient; NMSE-normalized mean squared error; MAE-mean absolute error

\section{Results and discussion}

The prediction performance of ANN-GA is evaluated by mean squared error (MSE), normalized mean squared error (NMSE), mean absolute error (MAE), minimum absolute error, maximum absolute error, and the linear correlation coefficient $(r)$ between the actual data and neural network outputs, and the results are shown in Table 4. From Table 4, it can be found that $\mathrm{r}$ is close to 1 , and NMSE is very small, showing that prediction performance of the GA-ANN model is optimal.

Table 4 GA-ANN performance

\begin{tabular}{cc}
\hline Performance & Number of neurons \\
NSE & 11.306 \\
MAE & 0.152 \\
Minimum absolute Error & 2.593 \\
Maximum absolute Error & 0.159 \\
$r$ & 5.870 \\
\hline
\end{tabular}

Table 5 shows the minimum MSE across all the 20 generations, the generation number at this minimum MSE, and the final MSE at the best fitness and average fitness of the generations for ANN-GA network. The average fitness is the average of the minimum MSE across all the networks which have different nodes in the hidden layer for a corresponding generation. Fig. 3 and Fig. 4 show that the best fitness and average fitness are improved with successive generations. In Table 6 , the outputs of ANN-GA are compared to the experimental data for saturates of VGO. Table 6 shows good agreement between the outputs of ANN-GA and the experimental data.

Table 5 Training result of ANN-GA model

\begin{tabular}{ccc}
\hline Optimization Summary & Best fitness & Average fitness \\
\hline Number of generation & 17 & 20 \\
Minimum MSE & 0.0029 & 0.0057 \\
Final MSE & 0.0029 & 0.0057 \\
\hline
\end{tabular}




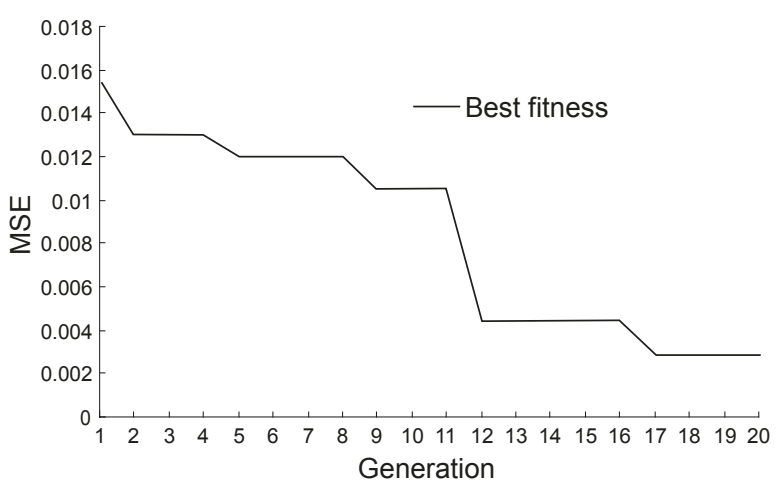

Fig. 3 The relationship of MSE vs. generation for best fitness of ANN-GA models

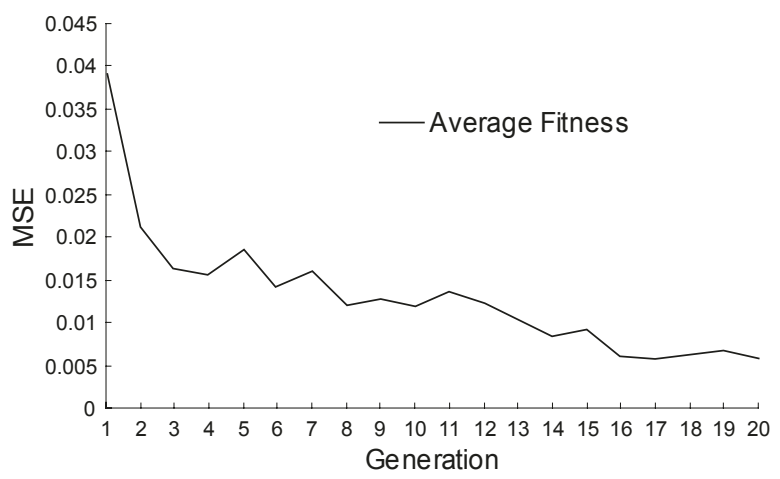

Fig. 4 The relationship of MSE vs. generation for average fitness of ANN-GA models

Table 6 The forecasting results of the ANN-GA model

\begin{tabular}{ccccccccc}
\hline NO. & $M$ & $D$ & $\eta$ & $n$ & $T_{\mathrm{b}}$ & $\mathrm{IO}$ & $\mathrm{AO}$ & $\mathrm{RE}$ \\
\hline 1 & 463 & 0.911 & 9.32 & 1.492 & 446 & 59.3 & 57.8 & -0.025 \\
2 & 425 & 0.858 & 4.26 & 1.463 & 316 & 78.5 & 79.2 & 0.009 \\
3 & 425 & 0.856 & 4.44 & 1.459 & 400 & 80.4 & 80.6 & 0.002 \\
4 & 425 & 0.872 & 6.24 & 1.485 & 400 & 85.8 & 82.1 & -0.043 \\
5 & 425 & 0.892 & 5.99 & 1.479 & 378 & 69.2 & 74.4 & 0.075 \\
6 & 463 & 0.849 & 5.33 & 1.455 & 360 & 84.2 & 90.1 & 0.069 \\
\hline
\end{tabular}

Notes: IO-output value of ANN-GA model for saturates content; AOexperimental data of saturates; RE-relative error of saturates content between the predicted result and the actual data

\section{Conclusions}

In this work, artificial neural networks based on a genetic algorithm are developed and used for predicting saturates of VGO. The number of neurons in the hidden layer, the momentum and the learning rates of back propagation algorithm are determined by using the GA algorithm. The inputs for the artificial neural networks model are five variables, namely, average boiling point, density at $20^{\circ} \mathrm{C}$, molecular weight, viscosity at $100{ }^{\circ} \mathrm{C}$ and refractive index at $70^{\circ} \mathrm{C}$. The study showed that the genetic algorithm could find the optimal number of neurons in the hidden layer, the momentum and the learning rates of the back propagation algorithm. In addition, the artificial neural networks model based genetic algorithm is tested and testing results are in good agreement with the experimental data. Compared with neural network models which are trained by trial-and-error approach, it can be found that the ANN-GA model is more efficient.

Moreover, recent studies show that the hydrogen-carbon ratio has significant influence on VGO saturates. Therefore, as long as data are available, further studies may be made by using ANN-GA models in future.

\section{References}

El-Hadi D and Bezzina M. Improved empirical correlation for petroleum fraction composition quantitative prediction. Fuel. 2005. 84(5): 611617

Funahashi K. On the approximate realization of continuous mappings by neural networks. Neural Networks. 1989. 2(3): 183-192

Goldberg D E. Genetic Algorithms in Search, Optimization and Machine Learning. Boston, MA: Addison-Wesley Longman Publishing Co. Lnc. 1989

Holland J. Adaptation in Natural and Artificial Systems. Ann Arbor, MI: University of Michigan Press. 1975

Hornik K, Stinchcombe M and White H. Universal approximation of an unknown mapping and its derivatives using multilayer feedforward networks. Neural Networks. 1990. 3(5): 551-560

Liu S B, Tian S B and Liu Y R et al. Study on the prediction of hydrocarbon type compositions of VGO based on its conventional characteristics. Petroleum Processing and Petrochemicals. 2007. 38 (9): 18-22 (in Chinese)

Michalewicz Z. Genetic Algorithms + Data Structures = Evolution Programs(third ed.). Berlin: Springer-Verlag. 1996

Nes K V and Westen H V. Aspects of the Constitution of Mineral Oils. New York: Elsevier. 1951

Nwadinigwe C A and Okoroji K A. Novel equations for quantitative hydrocarbon-type analysis of petroleum fractions. Fuel. 1990. (69): 340-343

Patuwo E, Hu M Y and Hung M S. Two group classification problem using neural networks. Decision Sciences. 1993. 24(4): 825-846

Riazi M R and Daubert T E. Prediction of the composition of petroleum fractions. Ind. Eng. Chem. Process Des. Dev. 1980.19(2): 289-294

Riazi M R and Daubert T E. Prediction of molecular-type analysis of petroleum fraction and coal liquids. Ind. Eng. Chem. Process Des. Dev. 1986. 25: 1009-1015

Rojas R. Neural Networks-A Systematic Introduction. Berlin, NewYork: Springer-Verlag. 1996

Sun R J, Wang S C and Zhao S Q. Study on prediction of sour vacuum gas oil (VGO) saturates by using artificial neural networks. Computers and Applied Chemistry. 2008. 25(11): 1393-1396 (in Chinese)

Waterman H I, Boehower C and Cornelissen J. Correlation between physical constants and chemical structure. New York: Elsevier. 1958

Xing B and Cheng Z. Studies on the prediction of hydrocarbon series composition of VGO. Qilu Petrochemical Technology. 2006. 34(2): 173-177 (in Chinese)

(Edited by Zhu Xiuqin) 